\title{
Analysis of the influence of defects on fatigue resistance of metallic components
}

\author{
Mirco D. Chapetti ${ }^{\mathrm{a}}$
}

Research Institute for Material Science (INTEMA), University of Mar del Plata - CONICET J.B. Justo, 4302 (7600) Mar del Plata, Argentina

\begin{abstract}
The influence of defect on fatigue resistance of components is carried out by using a propagation threshold approach to estimate the high cycle fatigue resistance of components from data of intrinsic properties of the material. The Chapetti's threshold curve for fatigue crack propagation is used, which includes the short crack regime. The approach accounts for the effective driving force applied to the crack as the difference between the total driving force applied to the component, $\Delta \mathrm{K}$, and the threshold for fatigue crack propagation of the material, $\Delta \mathrm{K}_{\mathrm{th}}$. Application to analyze the influence of plate thickness on fatigue resistance of welded joints is presented.
\end{abstract}

\section{Introduction}

The present work is concerned with the prediction of the fatigue strength of mechanical components by means of a fracture mechanics approach that includes the fatigue crack propagation threshold for both short and long cracks. The methodology estimates the threshold for fatigue crack propagation as a function of crack length, $\Delta \mathrm{K}_{\mathrm{th}}$, and the fatigue crack propagation rate as follows:

$$
\frac{d a}{d N}=C\left(\Delta K-\Delta K_{t h}\right)^{m} .
$$

Where $\Delta \mathrm{K}$ is the applied driving force. The following expression for the material threshold for crack propagation as a function of the crack length, $\Delta \mathrm{K}_{\mathrm{th}}$, is used [1]:

$$
\Delta K_{t h}=\Delta K_{d R}+\left(\Delta K_{t h R}-\Delta K_{d R}\right)\left[1-e^{-k(a-d)}\right]=Y \Delta \sigma_{t h} \sqrt{\pi a} \quad a \geq d
$$

where $\Delta \mathrm{K}_{\mathrm{dR}}$ and $k$ are given by:

$$
\Delta K_{d R}=Y \Delta \sigma_{e R} \sqrt{\pi d} \quad k=\frac{1}{4 d} \frac{\Delta K_{d R}}{\left(\Delta K_{t h R}-\Delta K_{d R}\right)} .
$$

In the case of a component with defects the crack initiation period is usually minimized, and the initial crack length for the crack propagation period will be given by the maximum crack-like defect.

\footnotetext{
${ }^{a}$ Corresponding author: mchapetti@fi.mdp.edu.ar
}

This is an Open Access article distributed under the terms of the Creative Commons Attribution License 4.0, which permits unrestricted use, distribution, and reproduction in any medium, provided the original work is properly cited. 


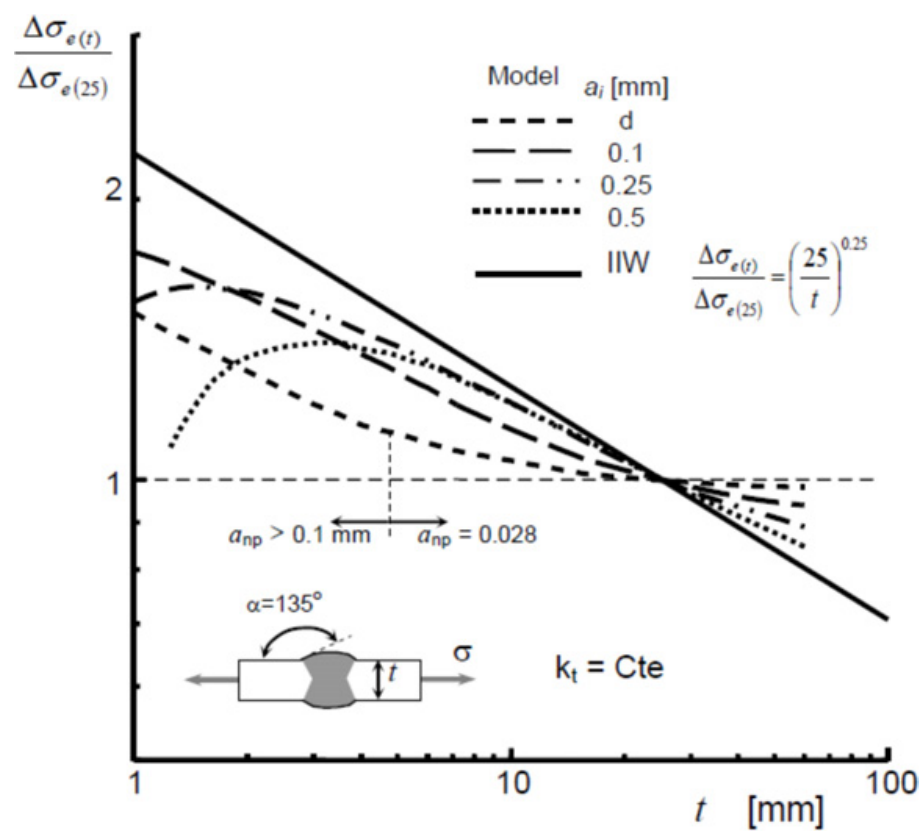

Figure 1. Influence of plate thickness on fatigue strength of butt-welds.

For the threshold condition the approach allows to estimate the non-propagating crack associated to the fatigue limit, which depends on the relative position of the applied driving force $\Delta K$ and the threshold for fatigue crack propagation $\Delta K_{\mathrm{th}}$. The maximum defect size allowable for that configuration can also be known, so that the approach can also analyze the defect size sensitivity of the analyzed configuration.

\section{Application example. Influence of plate thickness in weld joints}

It is well known that fatigue strength of welded joints decreases with increasing plate thickness. IIWrecommendation [2] suggests the following expression:

$$
\Delta \sigma_{t}=\Delta \sigma_{t 0}\left(\frac{t_{0}}{t}\right)^{n}
$$

where the reference thickness $t_{0}=25 \mathrm{~mm}$ and $n=0.25$, and $\Delta \sigma_{\mathrm{t} 0}$ is the fatigue strength for the reference thickness. Figure 1 shows estimated results of the fatigue strength defined at $10^{7}$ cycles as a function of the plate thickness for different initial crack lengths. Correction recommended by IIW for butt joint is also shown, extrapolated to thickness smaller than $25 \mathrm{~mm}$. Results show the expected trend in fatigue limit, but they also show notable differences in the influence of the initial crack length on fatigue limit of thin plates $(\mathrm{t}<10 \mathrm{~mm})$, and that extrapolations could overestimate fatigue strength for thickness smaller than $6 \mathrm{~mm}$. Results also show that an opposite trend could be found for thin plates $(\mathrm{t}<6 \mathrm{~mm})$ in the relationship between fatigue limit and plate thickness when initial crack length are longer than about $0.3 \mathrm{~mm}$. This opposite effect was observed experimentally by, for instance, Gustafsson [3] for non-load carrying attachments with 3 and $6 \mathrm{~mm}$ plate thickness.

The approach also allows understanding that the fatigue limit is given by $a_{\mathrm{i}}=d=0.028 \mathrm{~mm}$ for thickness greater than $6 \mathrm{~mm}$, but it is given by a non-propagating crack longer than $0.1 \mathrm{~mm}$ for thickness 
smaller than $6 \mathrm{~mm}$. The relative position between the applied driving force distribution and the material crack propagation threshold curve seems to define a thickness range below which the fatigue limit is given by a given non-propagating crack.

\section{References}

[1] M.D. Chapetti. Int J Fatigue 25, 12 pp.1319-26 (2003)

[2] A. Hobbacher, IIW Doc XIII-2151-07/XV-1254-07 (2007)

[3] M Gustafsson, West Midlands, EMAS Ltd. p. 205-24 (2002) 